„Bologna“ nachgezeichnet; es wird aufgezeigt, warum Wolfgang Clement „in der historischen Bewertung der deutschen Wirtschaftsminister schwerlich einen Spitzenplatz einnehmen“ (S. 158 f.) wird. Es ist nachzulesen, dass Herta Däubler-Gmelin in „nicht wenigen innerparteilichen Kontroversen polarisierte“ (S. 162) und selbstverständlich auch außerhalb der Partei (S. 170: „Hitler-Bush-Vergleich“) schadete. Über Hans Eichel heißt es, dass er ungeachtet vieler Anfeindungen „bis zur Ablösung des Kabinetts Schröders im Amt ausharrte“, sei „seiner Hartnäckigkeit, seinem Pflichtbewusstsein und seiner - arg strapazierten Loyalität zu Kanzler und Partei zuzuschreiben“ (S. 186). Gern liest man auch den Beitrag über Joschka Fischer und seinen Wandel vom „Straßenkämpfer“ (S. 195) zum Außenminister, der „in einer außergewöhnlichen innerparteilichen Kraftanstrengung seine eigene Partei an die realpolitischen Notwendigkeiten heran“ geführt hatte und „wesentlich zur Stabilisierung des deutschen Regierungssystems“ beitrug (S. 209). Selbst diejenigen, die es vielleicht schon gewusst haben, werden daran erinnert, dass der Rücktritt des „Paradiesvogels“ (S. 220) Bodo Hombach im Zusammenhang mit Oskar Lafontaines Rücktritt als Finanzminister stehen „dürfte“ (S. 219). Der Beitrag über die „grüne Allzweckwaffe“ (S. 224) Renate Elly Künast ist ein schöner Beleg dafür, mit welch großer Sorgfalt biographische Details zusammengetragen und anschaulich vorgetragen werden. Und weil die Biographien die Ministertätigkeit im Blick haben, vermisst der Leser im Beitrag über Ursula („Ulla“) Schmidt „geb. Radermacher" (offensichtlich die einzige Frau im Kabinett, die durch Heirat ihren Mädchennamen abgelegt hatte) nicht wirklich den Hinweis, womit sie als Studentin ihren Lebensunterhalt verdient hatte. Selbstverständlich bekommt der „Basta-Kanzler“ den umfangreichsten Artikel. Der Mitherausgeber Udo Kempfkennt und beherrscht die Literatur über Gerhard Schröder und zeichnet ein gelungenes Bild über seine Kanzlerschaft. Von ihr werden „Riester-Rente“ und „Agenda 2010“ (also der Umbau des Sozialstaates) lange in Erinnerung bleiben, genauso wie jenes Faktum, dass ausgerechnet unter Rot-grün die Bundeswehr in einen Krieg geschickt wurde (und nicht „zog“, wie Kempf, S. 338, schreibt).

Solche Wertungen und Bewertungen machen einmal mehr deutlich, dass auch wenn möglicherweise manche Biografien von Wissenschaftlern verfasst wurden, die der Partei des von ihnen behandelten Ministers nahestehen oder sogar angehören, diese sich verpflichtet fühlten, objektiv zu bleiben und auch gelegentliche Schattenseiten nicht zu verschweigen. So kann am Schluss zweierlei Lob stehen: Ein ehrliches Buch! Und: Mehr als nur ein Lexikon!

Michael F. Feldkamp

\title{
Kanzler und ihr Verhältnis zur Öffentlichkeit: wenig überzeugend bearbeitet
}

\section{Rosumek, Lars: Die Kanzler und die Medien. Acht Porträts von Adenauer bis Merkel, Campus- Verlag, Frankfurt am Main 2007, 325 Seiten, $€ 29,90$.}

Bis in die Gegenwart reichende Längsschnittanalysen haben einen ganz besonderen Charme: Sie schützen davor, aktuelle Entwicklungen für einmalig zu halten, kontextualisieren sie und können im besten Fall helfen, ihre Ursachen besser zu verstehen. Im Zusammenhang mit den Veränderungen politischer Kommunikation sind in den letzten Jahren eine ganze Reihe solcher Studien publiziert worden. Sie haben sich zum Beispiel mit den 
Veränderungen von Wahlkampfstrategien, Wahlwerbung, Wahlkampfberichterstattung der Medien oder dem relativen Einfluss verschiedener Faktoren auf individuelles Wahlverhalten beschäftigt. ${ }^{1}$ Der nach einem Studium der Kommunikationswissenschaft als PR-Berater tätige Lars Rosumek will mit seinem Buch einen weiteren Beitrag in diesem Bereich leisten. Er nimmt dabei das Verhältnis der bisherigen Kanzler zu den Medien und ihre Öffentlichkeitsarbeit in den Blick. Titel und Rückentext des bei Campus erschienenen und damit an ein breites, nicht nur wissenschaftliches Publikum gerichteten Bandes schrauben die Erwartungen hoch: Es werden acht Porträts der Kanzler und ihrer Verhältnisse zu den Medien versprochen und die „Aufdeckung“ der Tatsache, dass in Sachen PR „bereits vor 50 Jahren vieles aus den USA importiert wurde“. Leider werden die hochgesteckten Erwartungen nicht erfüllt. Dies hat vor allem vier Gründe.

Erstens liefert der Autor nicht - anders als es Klappentext, Vorwort und Einleitung suggerieren - spektakuläre Enthüllungen oder neue Ideen. Vielmehr stützt sich Rosumek neben einigen Interviews in erster Linie auf kommunikations- und politikwissenschaftliche Literatur. Allerdings ist er hier keineswegs auf dem 2006 beziehungsweise 2007 aktuellen Stand der Diskussion. So wurde das von ihm entworfene Bild einer simplen Amerikanisierungsthese zu diesem Zeitpunkt in der Kommunikations- und Politikwissenschaft schon lange nicht mehr vertreten (im Übrigen auch nicht in der zitierten Literatur). Rosumek baut also aus rhetorischen Gründen einen Scheingegensatz auf, der zumindest für die einschlägigen Wissenschaftsdisziplinen so nicht existiert. Dies ist umso ärgerlicher, als oftmals nicht deutlich wird, wo eigentlich seine Eigenleistung liegt, denn er verzichtet „zugunsten besserer Lesbarkeit“ auf einen „Großteil der ursprünglich aufgebotenen“ Literaturbelege. Dies macht es dem Leser zuweilen schwer zu entscheiden, wann der Autor schlicht die Ausführungen anderer wiedergibt, wann er sich auf seine Interviewpartner bezieht und wann es sich um eigene Gedanken, Ideen und Interpretationen handelt. Selbst wenn er zu Beginn eines Kapitels die Forschungslage oder einzelne Studien als ungenügend abqualifiziert, kann es sein, dass er sich im weiteren Verlauf absatzweise auf eben diese bezieht (ohne es hinreichend kenntlich zu machen).

Zweitens sind die Ausführungen zu den sieben Kanzlern und der derzeitigen Amtsinhaberin von sehr unterschiedlichem Umfang. Auf Ludwig Erhard und Kurt-Georg Kiesinger zusammen entfallen beispielsweise gerade einmal sechs Seiten, auf Konrad Adenauer dagegen 30. Dies ist zum einen der Tatsache geschuldet, dass zu manchen Kanzlern bereits recht viel gearbeitet und publiziert wurde, zu anderen dagegen nicht - was die Bedeutung der Sekundäranalyse nochmals klarstellt. Zum anderen konnte der Autor für Kiesinger und Erhard offenbar keine Zeitzeugen als Interviewpartner gewinnen - wieso und weshalb erfährt der Leser nicht. Mögliche alternative Informationsquellen, in denen man auf neue Erkenntnisse hätte stoßen können (etwa Archivmaterial oder die Medienberichterstattung) hat Rosumek nicht erschlossen. Er stützt sich dagegen auf Interviews mit Medienberatern, die im Original abgedruckt sind und die die interessantesten Seiten des Bandes bilden.

Drittens fehlt es dem Buch an einem analytischen Rahmen, der der Darstellung einen nachvollziehbaren roten Faden und mehr Struktur verliehen hätte. So wäre am Beginn beispielsweise eine Systematisierung von Einflussfaktoren hilfreich gewesen, die das Ver-

1 Einen Überblick liefert Carsten Reinemann, Wandel beschrieben - Wandel erklärt? Wahlkampfkommunikation im Langzeitvergleich, in: Gabriele Melischek / Josef Seethaler / Jürgen Wilke (Hrsg.), Medien- und Kommunikationsforschung im Vergleich, Wiesbaden 2007, S. 179 - 198. 
hältnis der Kanzler zu den Medien sowie ihre strukturellen und kommunikativen Maßnahmen im Rahmen der Öffentlichkeitsarbeit erklären könnten. Zu denken wäre hier etwa an die Ereignislage und die politische Situation auf Bundesebene, die Entwicklung des Mediensystems, die bestehenden Strukturen der Öffentlichkeitsarbeit sowie persönliche Faktoren.

Viertens finden sich auch einige sachliche Fehler und zumindest fragwürdige Aussagen. So wird beispielsweise behauptet, der Amtsbonus für die Kanzler in der Berichterstattung bestehe in ihrer besseren Bewertung (S. 47). Dies ist gerade nicht der Fall. Der Amtsbonus drückte sich bei früheren Wahlen in einer umfangreicheren und nicht in einer positiveren Berichterstattung aus. ${ }^{2}$ Kurios ist auch die unbelegte Feststellung, die Idee zur Gründung des Instituts für Demoskopie in Allensbach sei Anfang der 1950er Jahre in einem Kreis um Otto Lenz entstanden. Tatsächlich wurde das IfD Allensbach schon 1947 gegründet. $^{3}$

Insgesamt kann der Band von Lars Rosumek aus den genannten Gründen nicht überzeugen. Er macht aber zumindest deutlich, wo noch immer Lücken in der historischen Aufarbeitung des Verhältnisses zwischen Kanzlern und Medien bestehen.

Carsten Reinemann

2 Vgl. Jürgen Wilke / Carsten Reinemann, Die Normalisierung des Sonderfalls? Die Wahlkampfberichterstattung der Presse 2005 im Langzeitvergleich, in: Christina Holtz-Bacha (Hrsg.), Die Massenmedien im Wahlkampf. Die Bundestagswahl 2005, Wiesbaden 2006, S. 306 - 337.

3 Vgl. Elisabeth Noelle-Neumann, Die Erinnerungen, München 2006.

\section{Herausforderungen für die Parteien in Deutschland: flott - und schnell - formuliert}

Leif, Thomas: Angepasst und ausgebrannt: Die Parteien in der Nachwuchsfalle. Warum Deutschland der Stillstand droht, C. Bertelsmann Verlag, München 2009, 494 Seiten, $€ 22,95$.

Umschlaggestaltung und Titelgebung zeigen, dass Thomas Leif und der Bertelsmann-Verlag eine Neuauflage ihres Erfolges in den Buchhandlungen anstreben: 2006 erschien vom selben Autor „beraten und verkauft“ und wurde zum Sachbuch-Bestseller. Leif verteilte dort viele Schläge gegen Unternehmensberater, die aber oftmals am Ziel vorbeigingen: Verwendete Begriffe blieben unklar, Informationen wurden selektiv wahrgenommen und einseitig interpretiert - und das in einem selbstgerechten Ton, der auf der Welle der großen Kritik am Einsatz von Beratungsfirmen in Ministerien und insbesondere bei der Umstrukturierung der Bundesanstalt für Arbeit schwamm. Von der Politikwissenschaft ist das Buch daher zu Recht kaum beachtet worden.

Angesichts der fast identischen Gestaltung der Titelseite und der reißerischen Namensgebung ist daher bei ,angepasst und ausgebrannt“ zunächst Skepsis angebracht. Und tatsächlich findet man auch im Innern des Buches viele Ähnlichkeiten: flotte Formulierungen, Zitate aus internen „Geheimdokumenten“, reichlich Interviews mit Experten, die Reportage einer teilnehmenden Beobachterin - diesmal nicht zur Rekrutierung bei McKinsey, sondern beim CDU-Landesverband in Bremen. 